\title{
Experimental and Simulation Analysis of Effect of Fillet Radius of Micro Square Hole on Copper Sheets in Deep Drawing of Sheets
}

\author{
Tsung-Chia Chen, ${ }^{1}$ Ching-Min Hsu, ${ }^{1}$ and Cheng-Chi Wang ${ }^{2 *}$ \\ ${ }^{1}$ Department of Mechanical Engineering, National Chin-Yi University of Technology, \\ No. 57, Sec.2, Zhongshan Rd., Taiping Dist., Taichung 411030, Taiwan \\ ${ }^{2}$ Graduate Institute of Precision Manufacturing, National Chin-Yi University of Technology, \\ No. 57, Sec.2, Zhongshan Rd., Taiping Dist., Taichung 411030, Taiwan
}

(Received May 15, 2021; accepted August 19, 2021)

Keywords: micro deep drawing, springback, copper, material deformation history

In the design and fabrication of molds in the deep drawing process, the gap between the punch and the mold is extremely small. Obtaining a product of the correct size and shape necessitates the control of certain variables in the process, consideration of the problems of springback after load removal and the occurrence of cracks in the drawing process, and the estimation of the punch load. In this study, we focused on copper sheets and considered the influence of the scale effect and fillet radius of a micro square hole on thin sheets on the basis of an updated Lagrangian formulation (ULF) and finite element analysis. Sheet behavior was simulated using a micro-elastoplastic material model, the performance of which was compared with that of models involving conventional materials. Subsequently, the Dynaform LS-DYNA solver was used for simulation analysis, and pre- and post-processing were carried out to obtain the material deformation history, as well as to determine the thickness change distribution and material stress and prepare strain distribution maps. It was found that the scale effect of the sheet thickness influences the relation between the punch load and stroke, the distribution of thickness, the distribution of stress and strain, the maximum diameter of the flange hole, and the maximum flange height. Finally, the simulation results were compared with experimental results to confirm the accuracy of 3D finite element analysis of the elastoplastic deformation. The results show the influence of the fillet radius of the inner hole $(\mathrm{Br})$ for copper sheets on the drawing process: the punch load increases with increasing $\mathrm{Br}$, but the minimum thickness of the formed flange decreases as $\mathrm{Br}$ increases. The maximum principal stress/strain and the flange height increase as $\mathrm{Br}$ increases. The findings serve as a valuable reference for the design and processing of micro deep drawing.

\section{Introduction}

In recent years, in response to the growing demand for miniaturization, the production of sheet-formed products has experienced substantial growth. Sheet forming technology can be classified as drawing, stretch-flanging, and bending. During the forming process, insufficient

*Corresponding author: e-mail: wcc@ncut.edu.tw https://doi.org/10.18494/SAM.2021.3581 
formability or improper parameter setting may generate defects, such as fractures and excessive local thinning or wrinkling, which may negatively affect the precision of the workpiece. Life and yield must be compensated for in the mold design stage. The miniaturization of the sheet is influenced by the scale effect; thus, the parameter control differs from the general forming conditions, such as the surface roughness of the element, the friction during the forming process, the plastic flow stress of the material, and springback. Therefore, accurate prediction and control of the parameters in the micro-forming process are essential. ${ }^{(1-4)}$

Sheet metal micro-forming is one of the key processing techniques in the manufacturing industry. Sheet metal forming is a manufacturing technique used to process products with specific geometric shapes by varying the relative displacement between a metal sheet and a die. Sheet metal forming is currently divided into three basic models, ${ }^{(5)}$ namely, drawing, stretchflanging, and bending. A metal sheet bears different forces for different forming models; thus, various formability tests are utilized to evaluate the formability of sheet metal in an actual stamping. In the forming process, a metal sheet could exhibit fracture, excessive local thinning, or wrinkling because of unfavorable formability or poor setting of the processing parameters, and this problem must be explored by researchers and experts. Square hole-flanging is a type of sheet metal drawing, ${ }^{(6)}$ and the products can be applied to components, such as industrial products of automobiles, inner sheets of airplanes, pipe connections, and general household appliances. In general sheet metal forming, the processing parameters are acquired by trial and error or from experience in general die factories; thus, the data cannot be controlled for process design. If square hole-flanging could be presimulated to optimize the processing parameters as an alternative to trial and error in general die factories, the labor, time, and cost spent resolving errors could be reduced.

Kawka and Makinouchi ${ }^{(7)}$ proposed degenerated shell elements and combined them in a static explicit finite element equation to compare distinct integration rules, including complete integration, simplified integration, selective reduced integration, the assumed strain field, and the stable matrix rule. When $\mathrm{Leu}^{(8)}$ discussed hole-flanging with the elastic-plastic finite element method (FEM), the stress borne by the hole periphery was assumed as a single circular drawing stress so that the destructive model was simplified into a simple drawing test; moreover, an if-statement composed of the stress concentration factor, work hardening index, and orthotropic coefficient was deduced for the uniaxial drawing test to judge the hole edge necking of a hole-flange work. Takuda and Hatta ${ }^{(9)}$ analyzed sheet metal forming with the rigid-plastic FEM and utilized ductile fracture criteria to estimate the forming limit of commercial zircon sheet metal in the deep drawing process. The results showed that higher drawability of zircon sheet metal could be achieved and the location of fracture could be predicted by a combination of a finite element analysis program and ductile fracture criteria. Kumagai et al. ${ }^{(10)}$ studied the thinning in hole-flanging with $\mathrm{Al} / \mathrm{Cu}$ alloy sheet metal, where a cone punch with a cone angle of $45^{\circ}$ was used for the hole-flanging test. By comparing the FEM simulation results with the experimental results, they found that the rigid-plastic FEM could effectively predict the forming process and the final shape of the alloy sheet metal in the hole-flanging. Worswick and Finn ${ }^{(11)}$ simulated the forming of a round hole flange, square hole flange, and $\mathrm{Z}$ hole flange with the explicit dynamic FEM. They combined the forming limit diagram with the von Mises, Hill, and 
Barlat yield criteria to discuss the forming limit of sheet metal. Huang and Chien ${ }^{(12)}$ analyzed the forming limit of a cylindrical punch radius in hole-flanging by numerical analyses; the effect of the punch contour radius on the forming limit was not obvious, but the effect on the punch load was large. Leu et al. ${ }^{(13)}$ compared the maximal punch load in hole-flanging with various geometric shapes of the punch (cone, hemisphere, and cylinder) while changing some of the processing parameters in a numerical simulation. The experimental results showed that the maximal punch load in forming was for the cylindrical punch, with a smaller strain hardening index, a larger friction coefficient, a larger punch angle, and a larger forming load for the cone punch.

In this study, a square inner hole blank was stretched and formed with a square punch such that the blank adhered to the arc corner of the punch and bend, forming a square column shape. The deformation behavior of a flange with a micro square hole in stretch forming is complex, and continuous simulation and analysis are required to obtain a finished product with an accurate size and shape. Dynaform finite element analysis software was used to simulate the stretch forming of copper sheets. Experiments were performed to confirm and analyze the results, specifically to determine whether Dynaform can be accurately applied to stretch forming, to promote its use in relevant factory procedures, and to provide stretch forming parameters and a mold design for reference.

\section{Theoretical Analysis}

\subsection{Basic hypothesis and evolution of principle}

Several assumptions are made for the blank subjected to elastoplastic deformation considered in this study.

- The material is homogeneous.

- The material is planar anisotropic.

- The material satisfies Hooke's Law in the elasticity area.

- Dies are regarded as rigid bodies.

- The effect of residual stress is not considered in the material forming.

- The effect of temperature is not considered in the material forming.

A governing equation suitable for various metal forming processes is deduced from the stress-stress rate relationship in metal material deformation, the updated Lagrangian formulation (ULF) under finite deformation, and the material composition. The Jaumann differential of the Cauchy stress is used for the stress rate of the constitutive equation; thus, the updating principle of the virtual work derived by the Lagrangian can omit the volume function, and the equation can be revised as follows: ${ }^{(14)}$

$$
2 \int_{V^{E}}\left(\stackrel{\circ}{\sigma}-2 \sigma_{i k} \dot{\varepsilon}_{k j}\right) \dot{\delta} \dot{\varepsilon}_{k j} d V+\int_{V^{E}} \sigma_{j k} L_{i k} \delta L_{i j} d V=\int_{S_{f}} \dot{f} \delta v_{i} d S
$$

Here, $\stackrel{\circ}{i j}_{i}$ is the Jaumann differential of the Cauchy stress, $\sigma$ is the Cauchy stress tensor, $\dot{\varepsilon}$ is the 
Cauchy strain rate tensor, $L$ is the velocity gradient matrix, $v_{i}$ is the velocity, $\mathrm{d} S$ is the differential surface area after deformation, $f$ is the rate of the unit surface force, and $\mathrm{d} V$ is the differential volume after deformation.

The following common equations in the FEM are also included.

$$
\begin{aligned}
& \{v\}=[N]\{\dot{d}\} \\
& \{\dot{\varepsilon}\}=[B]\{\dot{d}\} \\
& \{L\}=[E]\{\dot{d}\}
\end{aligned}
$$

Here, $[N]$ is the shape function, $\{\dot{d}\}$ is the nodal velocity, $[B]$ is the strain rate-velocity matrix, and $[E]$ is the velocity gradient-velocity matrix. After finite element discretization, the rigid governing equation for large deformation is

$$
[K]\{\Delta u\}=\{\Delta F\}
$$

where

$$
[K]=\sum_{\langle E\rangle} \int_{V^{E}}[B]^{T}\left(\left[C^{e p}\right]-[Q]\right)[B] d V+\sum_{\langle E\rangle} \int_{V^{E}}[E]^{T}[Z][E] d V
$$

$[K]$ is generally regarded as the overall elastoplastic stiffness matrix, $\{\Delta u\}$ is the nodal displacement increment, $\{\Delta F\}$ is the nodal force increment, $[Q]$ and $[Z]$ are stress-modified matrices, and $\left[C^{e p}\right]$ is the elastoplastic stress-strain matrix.

\subsection{Scale factor for sheet metal micro-forming process}

The size effect can be neglected when the thickness of the sheet is larger than $1.0 \mathrm{~mm}$, whereas the size effect is crucial when the thickness is less than $1.0 \mathrm{~mm}$. The thickness of the metal sheet is in the micron range in the micro-forming process; therefore, the size effect makes the traditional material model unsuitable for the micro-forming process. Consequently, a new material model must be established for the micro-forming process. The traditional Swift material model (without considering the size effect) was first applied in this study:

$$
\bar{\sigma}=K\left(\varepsilon_{0}+\bar{\varepsilon}_{p}\right)^{n}
$$

The applied sheet metal thickness was $50 \mu \mathrm{m}$, and this thickness is necessary to consider the size effect. The thickness of the sheet metal was considered in the traditional material model to modify the stress-strain relations. Consequently, Eq. (7) was amended as follows: ${ }^{(15)}$ 


$$
\bar{\sigma}(t, \bar{\varepsilon})=a K e^{b t}\left(\varepsilon_{0}+\bar{\varepsilon}_{p}\right)^{n\left(c e^{d t}-1\right)},
$$

where $a, b, c$, and $d$ are the correction values and $t$ is the sheet thickness.

The values for $a, b, c$, and $d$ obtained from the research of $\mathrm{Liu}^{(16)}$ were substituted into Eq. (8) to give

$$
\bar{\sigma}(t, \bar{\varepsilon})=0.73667 K e^{0.3152 t}\left(\varepsilon_{0}+\bar{\varepsilon}_{p}\right)^{n\left(1.0106 e^{-0.01029 t}-1\right)} .
$$

Finite element analysis was conducted for the modified material model in Eq. (9) in this study. Experiments were further implemented to verify the validity of the modified material model in Eq. (9).

\section{Numerical Analysis and Experiments}

The shell elements of the four nodes of a quadrangle are applied to deduce the stiffness matrix. These elements are processed with CAD, and mash segmentation is performed on the established blank shape for the numerical analysis in the elastoplastic 3D finite element program. The simulation results are output to CAD for analysis, and the results are displayed as a deformation diagram and a stress-strain distribution. The coefficient of friction, sheet thickness, and punch fillet radius are changed as parameters to discuss the relation between the punch load and punch stroke, the distribution of the maximal stress and maximal strain, and the thickness distribution.

\subsection{Numerical simulation analysis process}

The process of numerical simulation is as follows:

1. Draw 3D models of molds and blanks through blank size engineering (BSE) and die face engineering (DFE) design.

2. Mesh the 3D model and perform preprocessing in the file format specified by Dynaform.

3. Use Dynaform to set the process parameters of the mold and sheet data files and conduct numerical analysis with the LS-DYNA program.

4. Use Dynaform to postprocess the calculations and the analytical results, then output them for examination using graphics and animation programs.

5. Compare the data obtained from the numerical analysis and the experiment through computer integration.

6. Analyze the procedure through the process parameters, i.e., the fillet radius and the diameter of the inner hole of the blank and the fillet of the punch.

The friction coefficient $\mu$ of 0.1 set in the simulation was close to the actual friction value obtained. ${ }^{(17,18)}$ The simulation results for punch load, punch stroke, and stress and strain were output to Dynaform for postprocessing and subsequent analysis. During the postprocessing, the 
deformation history diagram was visualized, as were the stress and strain distribution diagrams for the punch stroke in each stage. These data can serve as a reference for mold design and drawing procedures to determine the probability of cracks or wrinkles and reduce the high financial and time costs associated with the conventional trial-and-error method.

\subsection{Experiments}

Because the mold gap in this experiment was extremely small and processing was not easy, micro-discharge machining technology was employed, reducing the processing time and the cost and fulfilling the accuracy requirements. Figure 1(a) and Table 1 respectively show the die and the size parameters in the micro hole-flanging; Fig. 1(b) and Table 2 respectively show the copper sheet and the size parameters in micro hole-flanging. The mechanical properties and material parameters of copper are shown in Table 3.

\subsubsection{Forming process of flange with micro square hole for copper sheets}

Table 4 shows the stroke parameters in the five stages of micro hole-flanging. In the drawing process, the major deformation of the sheet metal occurs in the contact area along the punch

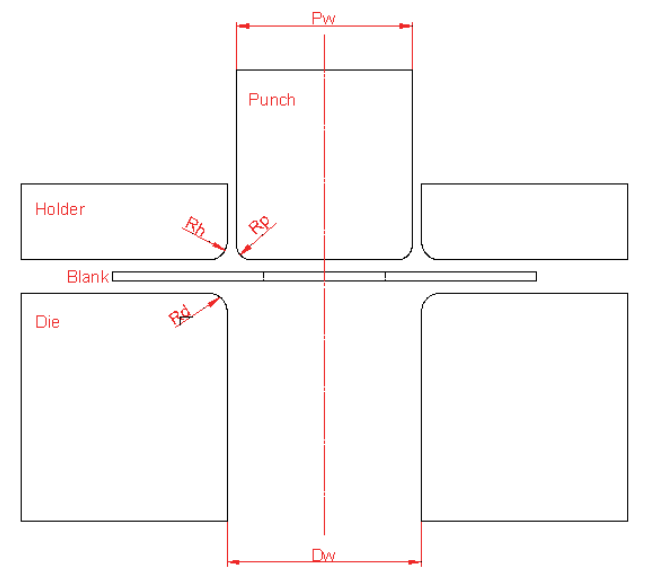

(a)

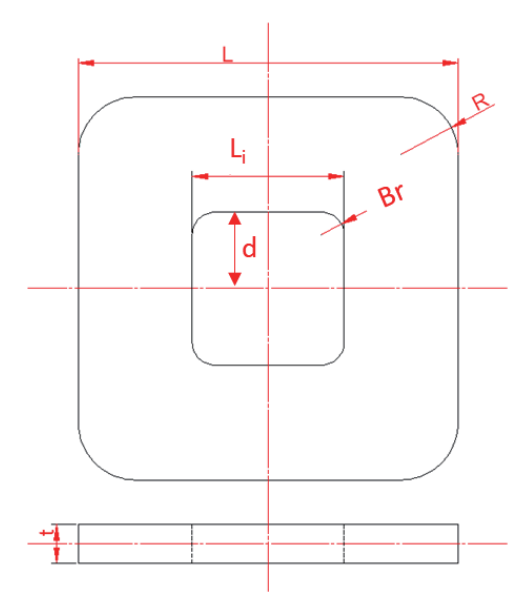

(b)

Fig. 1. (Color online) (a) Stretch module of flange with micro square hole and (b) copper sheet with micro square hole.

Table 1

Relevant parameters of flange mold (unit: $\mathrm{mm}$ ).

\begin{tabular}{lccc}
\hline & Model 1 & Model 2 & Model 3 \\
\hline$P_{W}$ & 10.00 & 5.00 & 2.50 \\
$D_{W}$ & 10.44 & 5.22 & 2.61 \\
$R_{p}$ & 1.00 & 0.50 & 0.25 \\
$R_{d}$ & 1.20 & 0.60 & 0.30 \\
\hline
\end{tabular}


Table 2

Parameters related to dimensions of flange material (unit: $\mathrm{mm}$ ).

\begin{tabular}{lccc}
\hline & Model 1 & Model 2 & Model 3 \\
\hline$L$ & 20 & 10 & 5 \\
$L_{i}$ & 9 & 4.5 & 2.25 \\
$R$ & 5 & 2.5 & 1.5 \\
$B r$ & 1.6 & 0.8 & 0.4 \\
$t$ & 0.2 & 0.1 & 0.05 \\
\hline
\end{tabular}

Table 3

Mechanical properties and material parameters of copper.

\begin{tabular}{lcccccc}
\hline Material & $E(\mathrm{GPa})$ & $v$ & $\sigma_{y}(\mathrm{MPa})$ & $K(\mathrm{MPa})$ & $n$ & $\varepsilon_{0}$ \\
\hline Model 1 & 26500 & 0.3 & 886.1 & 1160 & 0.81 & 0.42 \\
Model 2 & 19800 & 0.3 & 585.12 & 988.9 & 0.7 & 0.47 \\
Model 3 & 25254 & 0.3 & 224.6 & 565.8 & 0.207 & 0.011 \\
\hline
\end{tabular}

$E$ : Young's modulus; $v$ : Poisson's ratio; $\sigma_{y}$ : yielding stress.

Table 4

Relevant stroke parameters of flange (unit: $\mathrm{mm}$ ).

\begin{tabular}{lccr}
\hline Step & Model 1 & Model 2 & Model 3 \\
\hline S1 & 0.00 & 0.00 & 0.000 \\
S2 & 1.75 & 0.75 & 0.375 \\
S3 & 3.00 & 1.50 & 0.750 \\
S4 & 5.25 & 2.25 & 1.125 \\
S5 & 7.00 & 3.00 & 1.500 \\
\hline
\end{tabular}

corner and along the horizontal arc angle of the die, which are the drawing deformation areas. The punch bottom comes in contact with the sheet metal when the stroke moves from S1 to S2 and S3, and the sheet metal gradually deviates from the punch bottom with increasing stroke until the sheet metal in contact with the punch corner undergoes drawing deformation. When the stroke moves to $\mathrm{S} 3$, the sheet metal is completely pierced and the punch undergoes continuous displacement in the $-Z$ direction. From the deformation of the elements, the area of the sheet metal in contact with the punch corner induces larger circular drawing stress, which is gradually released when the stroke moves to S4.

\subsubsection{Experimental setup}

Figure 2(a) shows the die components, where the punch has a hierarchical design to enhance its rigidity. Tool steel (SKD11) is utilized for the die to improve its rigidity and wear resistance. Figure 2(b) displays the copper sheets before micro hole-flange drawing. 


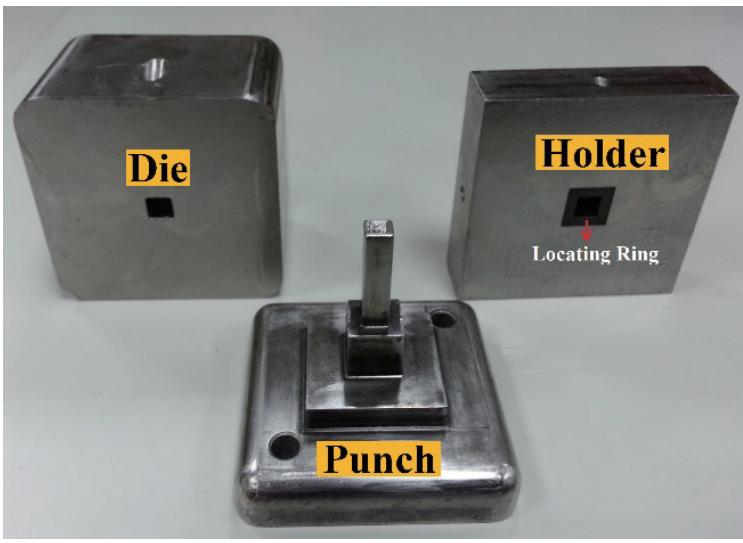

(a)

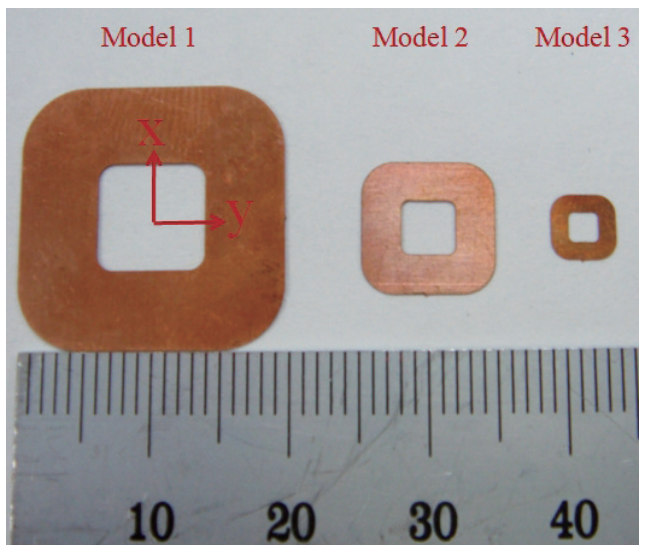

(b)

Fig. 2. (Color online) (a) Key components of mold and (b) C1100 copper applied in this experiment before drawing.

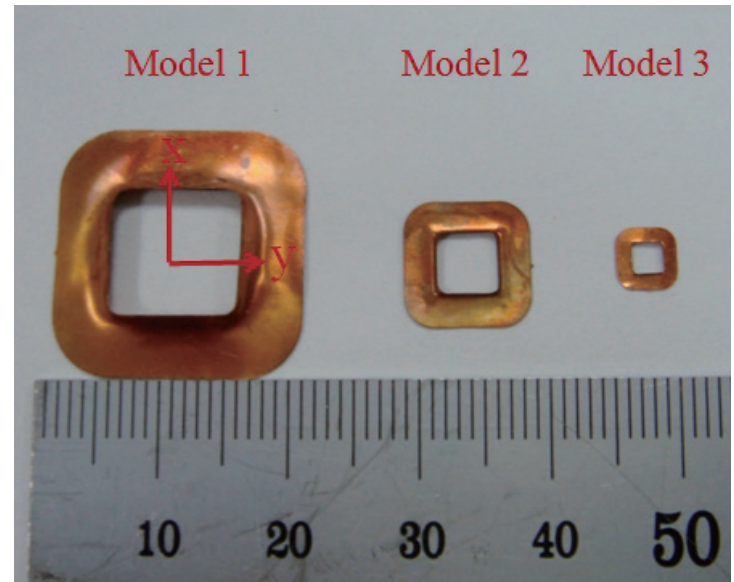

Fig. 3. (Color online) Finished micro square hole flange of C1100 copper sheet after punching and rebound.

\section{Results and Discussion}

\subsection{Verification of experiments and simulations}

Figure 3 shows the finished micro square hole flange after punching and rebound.

Figure 4 shows a comparison of the experimental and simulated loads of strokes during drawing. The experimental punch load is slightly larger than the simulated value. For a copper sheet, the maximum load is $148 \mathrm{~N}$ for Model 1 and the value decreases to $37.5 \mathrm{~N}$ as the sheet thickness decreases to $0.1 \mathrm{~mm}$. The upward trend of the load is also more moderate, mainly 


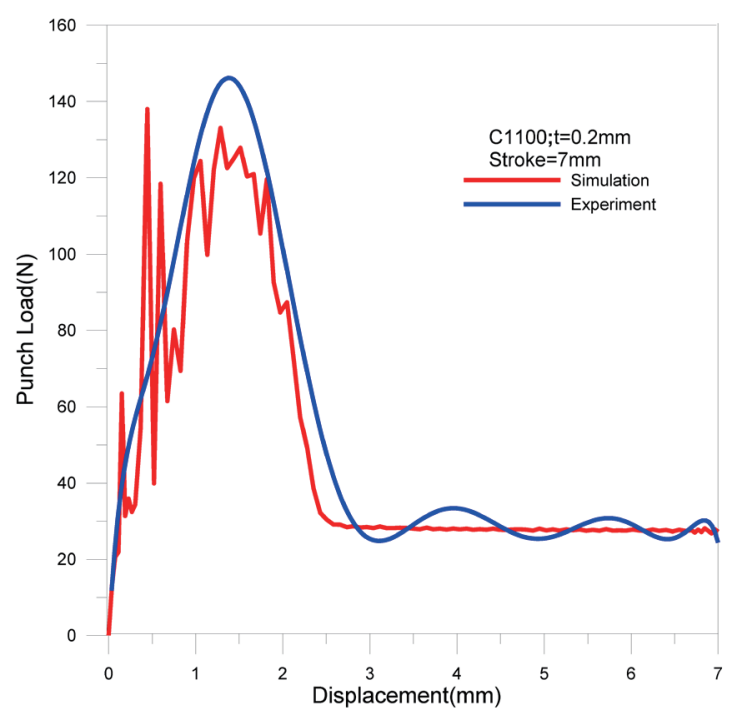

(a)

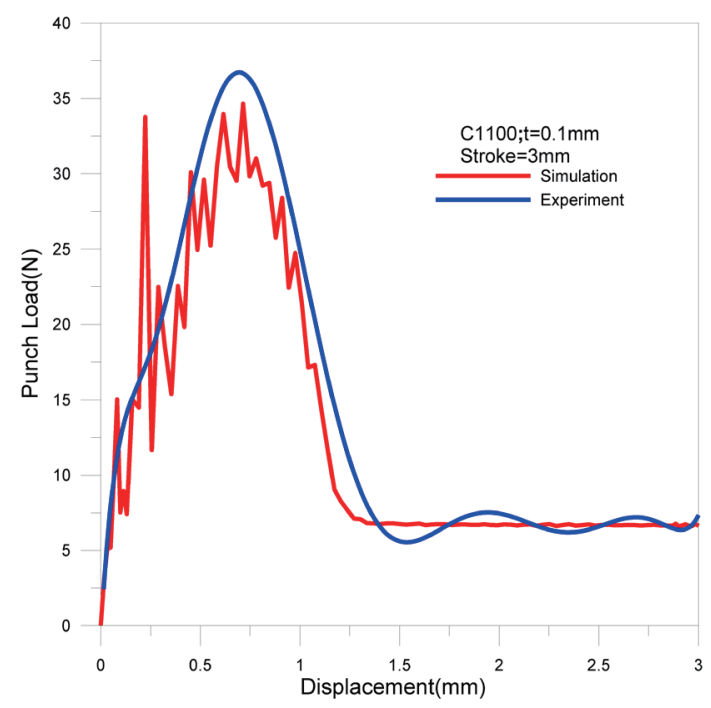

(b)

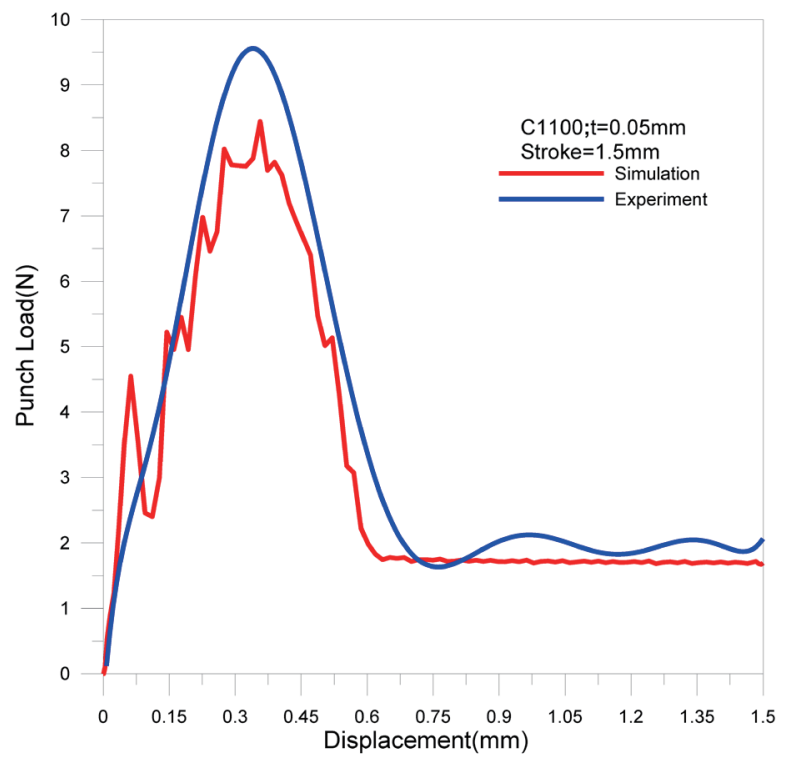

(c)

Fig. 4. (Color online) Comparison of experimental and simulated loads of strokes during drawing process: (a) Model 1; (b) Model 2; and (c) Model 3.

because copper is an fcc structure and its sliding of atoms is greater than that of bcc stainless steel, resulting in copper having less rigidity.

Figure 5 shows a comparison of the thickness at the location of $0^{\circ}$ for the copper sheet. From the difference in thickness, the accuracy of the contour curve, i.e., the difference between the theoretical value and the actual value, can be found. The difference is small for all three models: the maximum differences for Models $1-3$ are about $0.005,0.01$, and $0.015 \mathrm{~mm}$, respectively. 


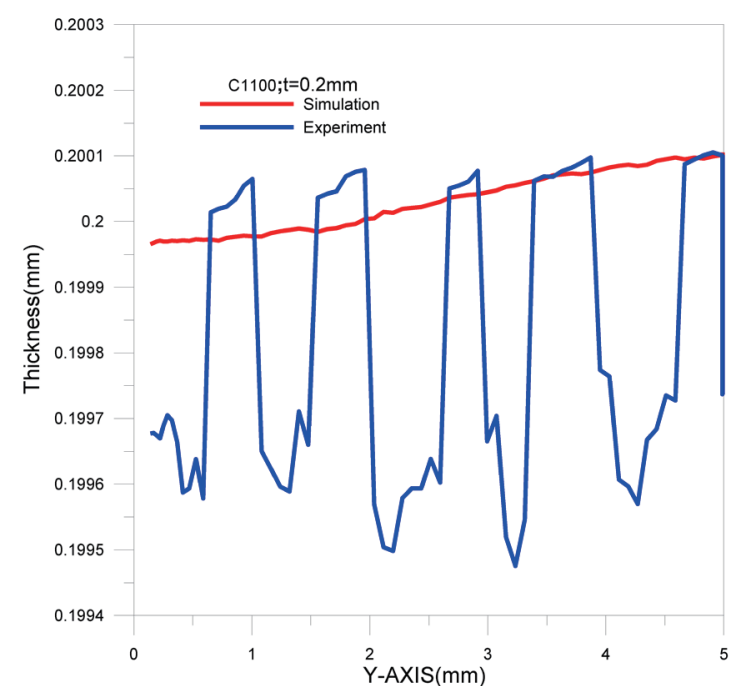

(a)

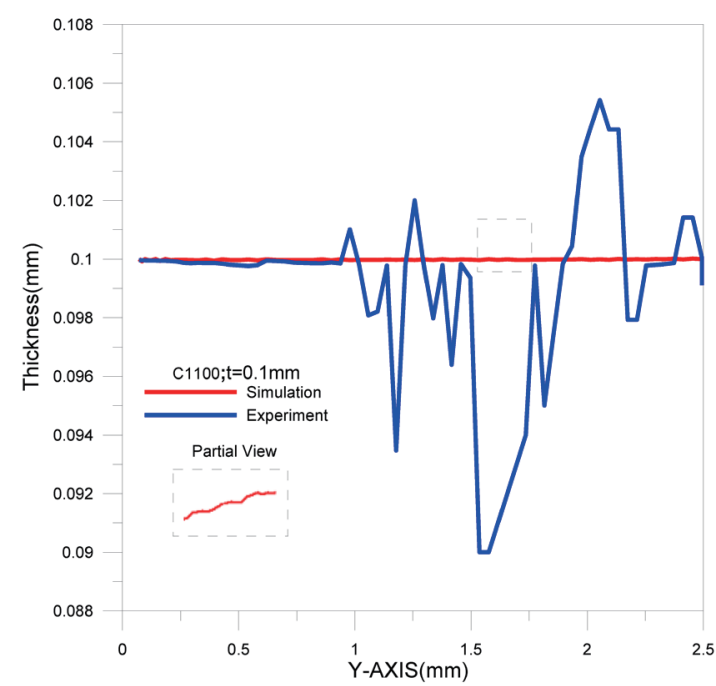

(b)

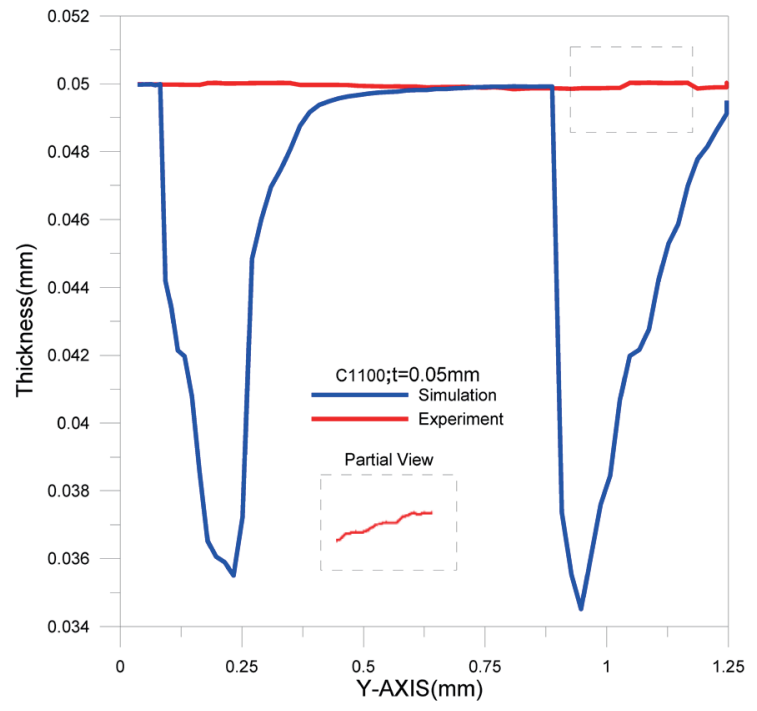

(c)

Fig. 5. (Color online) Comparison of thickness at location of $0^{\circ}$ for copper sheet: (a) Model 1; (b) Model 2; and (c) Model 3.

Figure 6 presents a comparison of the thickness at the location of $45^{\circ}$ for the copper sheet. As above, from the difference in thickness, the accuracy of the contour curve can be found. Again, the difference is small for all three models, with maximum differences of $0.006,0.001$, and 0.006 $\mathrm{mm}$ for Models $1-3$, respectively.

\subsection{Influence of fillet radius of inner hole for copper sheets on drawing process}

The drawing process was analyzed for different values of the fillet radius of the inner hole for the copper sheet. The radius of the fillet of the inner hole of the blank, denoted by $\mathrm{Br}$, is shown in 


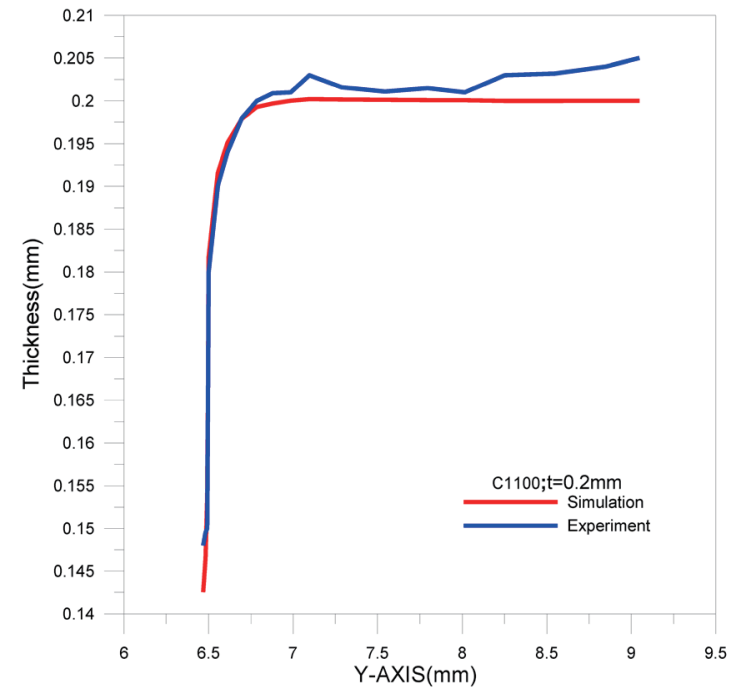

(a)

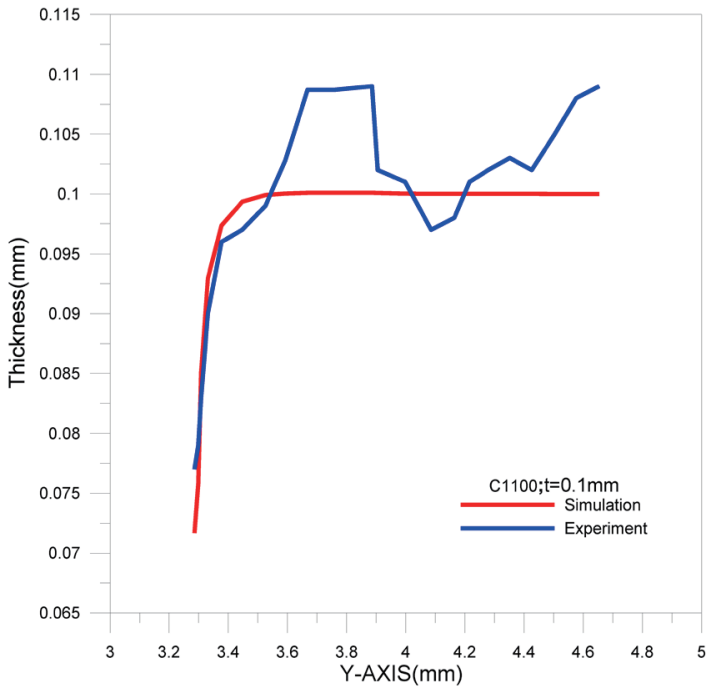

(b)

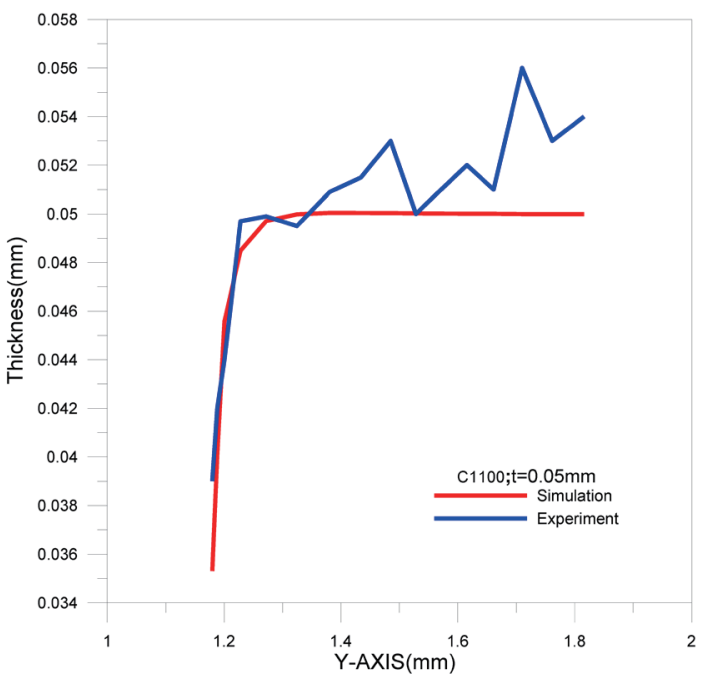

(c)

Fig. 6. (Color online) Comparison of thickness at location of $45^{\circ}$ for copper sheet: (a) Model 1; (b) Model 2; and (c) Model 3.

Table 5

Fillet radius and related parameters of inner hole for copper sheet (unit: $\mathrm{mm}$ ).

\begin{tabular}{lllllllc}
\hline Model No. & \multicolumn{7}{c}{$B r$} \\
Model 1 & 1.2 & 1.4 & 1.6 & 1.8 & 2 & 0.2 & Final stroke \\
Model 2 & 0.4 & 0.6 & 0.8 & 1.0 & 1.2 & 0.1 & 3 \\
Model 3 & 0.2 & 0.3 & 0.4 & 0.5 & 0.6 & 0.05 & 1.5 \\
\hline
\end{tabular}

Table 5. The material parameters are all corrected by the scale factor, and all materials are regarded as isotropic. 


\subsubsection{Relationship between load and stroke of punch with different $\mathrm{Br}$ values}

Figure 7 shows the relationship between the load and stroke of the punch for different values of $\mathrm{Br}$. The figures show that the load on the punch increased with an increase in $\mathrm{Br}$, which was mainly caused by an increase in the area of contact between the punch and the sheet.

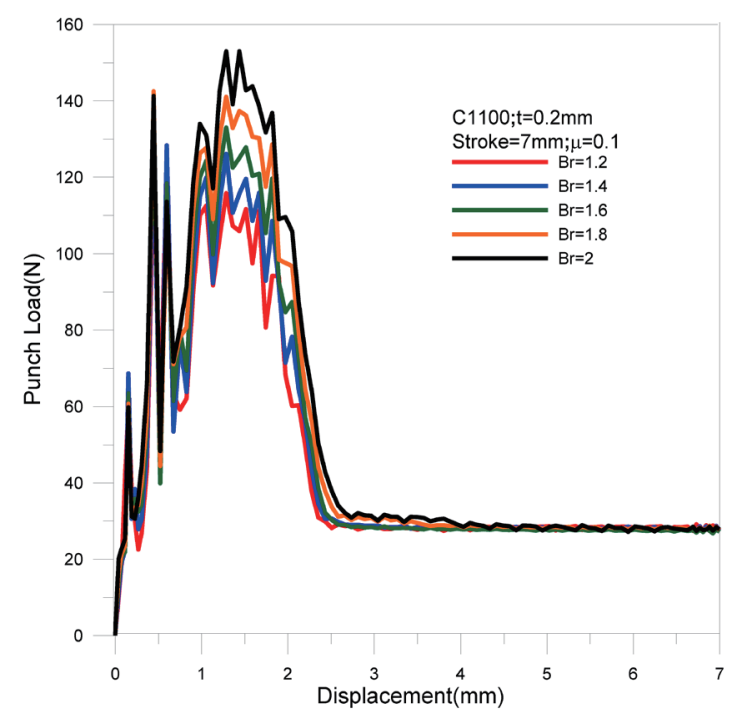

(a)

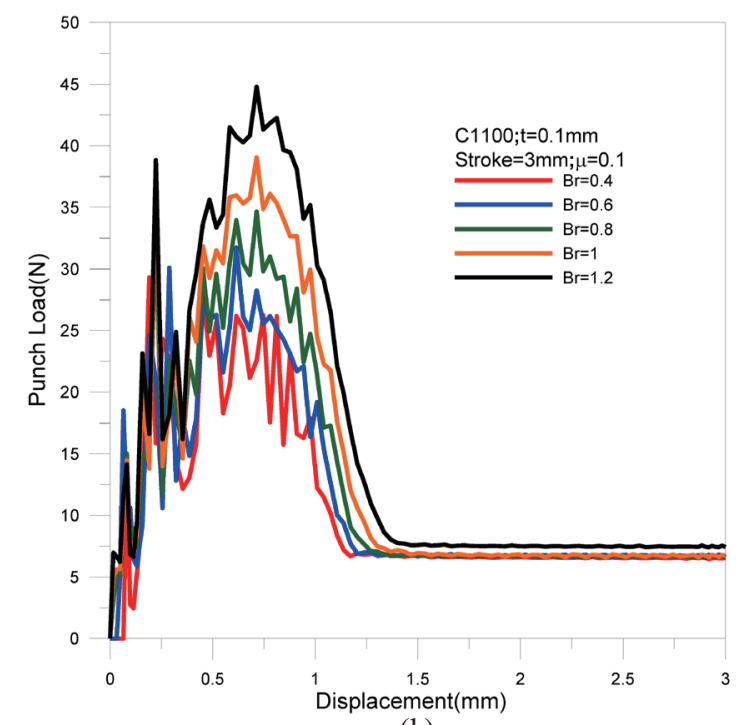

(b)

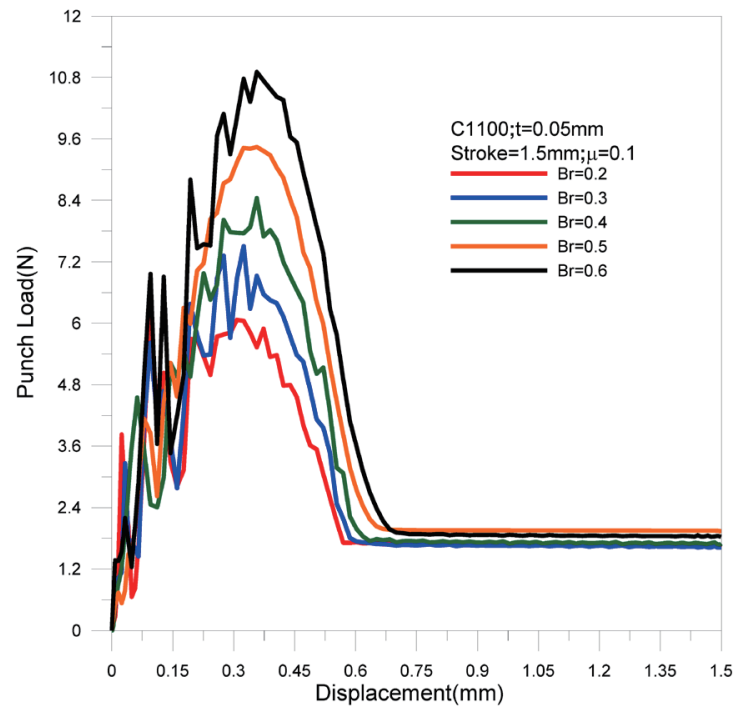

(c)

Fig. 7. (Color online) Relationship between load and stroke of punch with different values of Br: (a) Model 1; (b) Model 2; and (c) Model 3. 


\subsubsection{Relationship between fillet radius of inner hole and minimum thickness of flange}

Figure 8 shows the relationship between $\mathrm{Br}$ and the minimum thickness of the flange. This relationship was observed with all three models and was mainly due to the better formability at the corners of the square hole with an increase in the value of Br. However, the flange will also be higher. In actual production, the deformation and thickness of the sheet should be as uniform as possible. The average values of the minimum thickness for Models 1-3 are 0.1415, 0.071, and $0.03485 \mathrm{~mm}$, respectively.

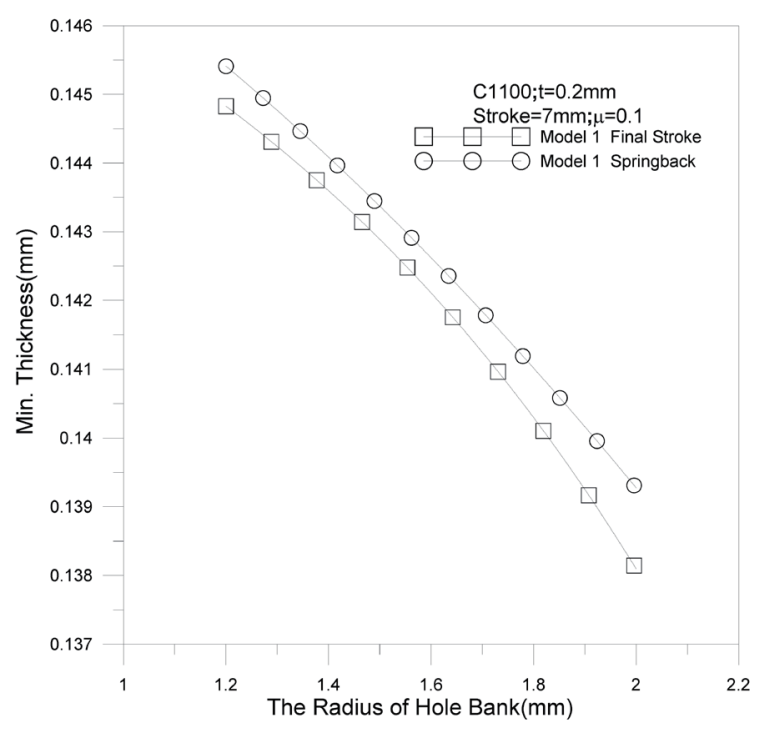

(a)

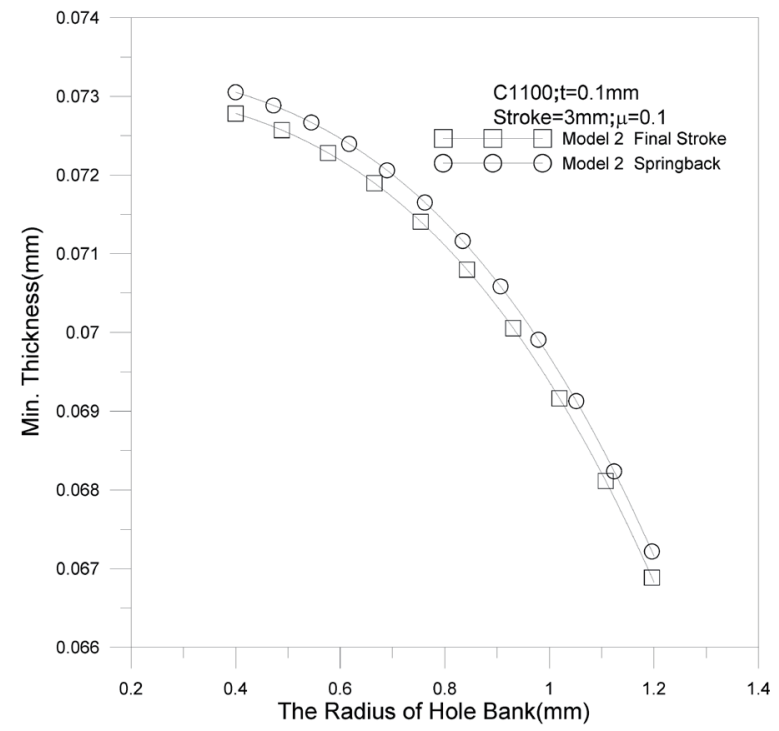

(b)

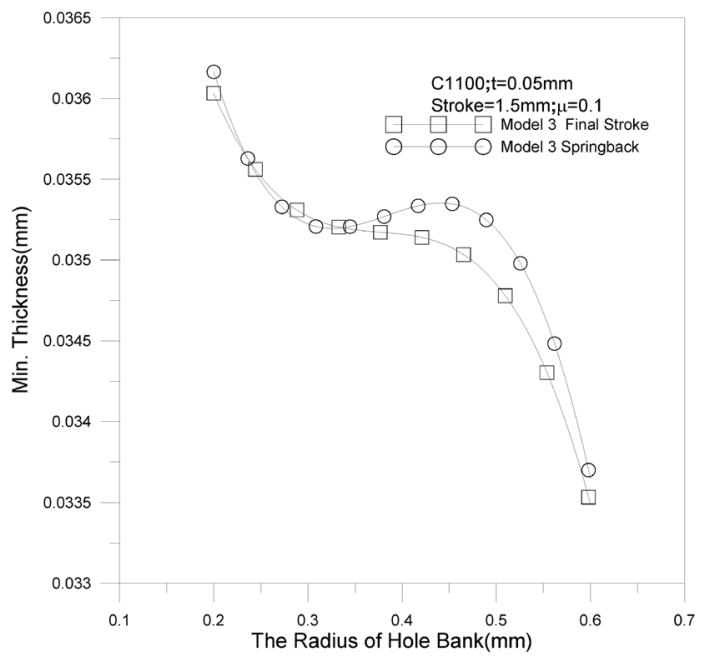

(c)

Fig. 8. Relationship between Br and minimum thickness of flange: (a) Model 1; (b) Model 2; and (c) Model 3. 


\subsubsection{Relationship between fillet radius of inner hole and maximum principal stress/strain}

The maximum stress and strain occur in the contact area between the sheet and the corner of the punch. The maximum principal stress increases as the value of $\mathrm{Br}$ increases, as shown in Fig. 9. This is because the larger value of $\mathrm{Br}$ induces a larger internal force in the material during forming, which causes the stress to rise.

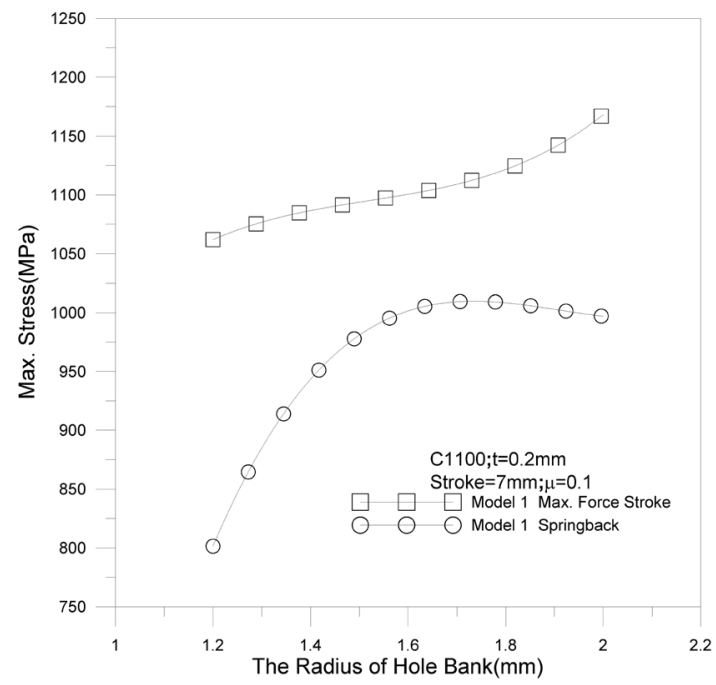

(a)

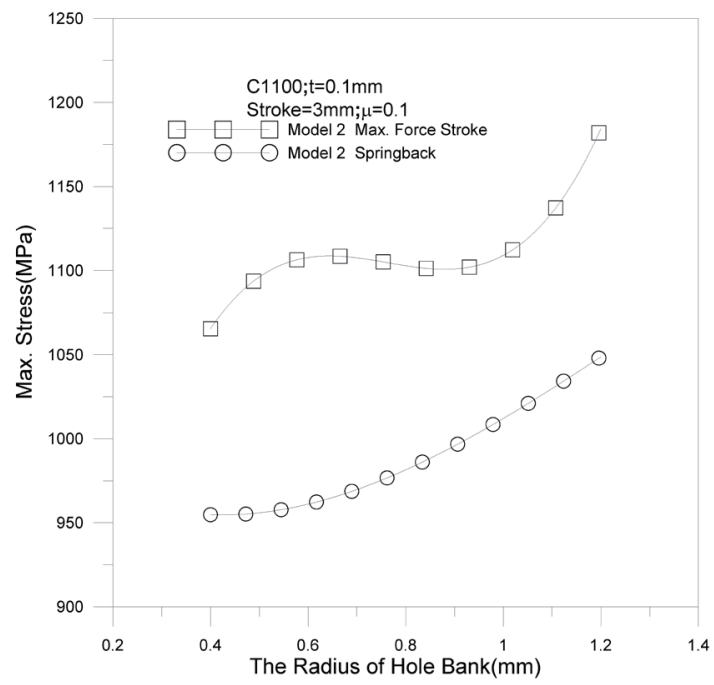

(b)

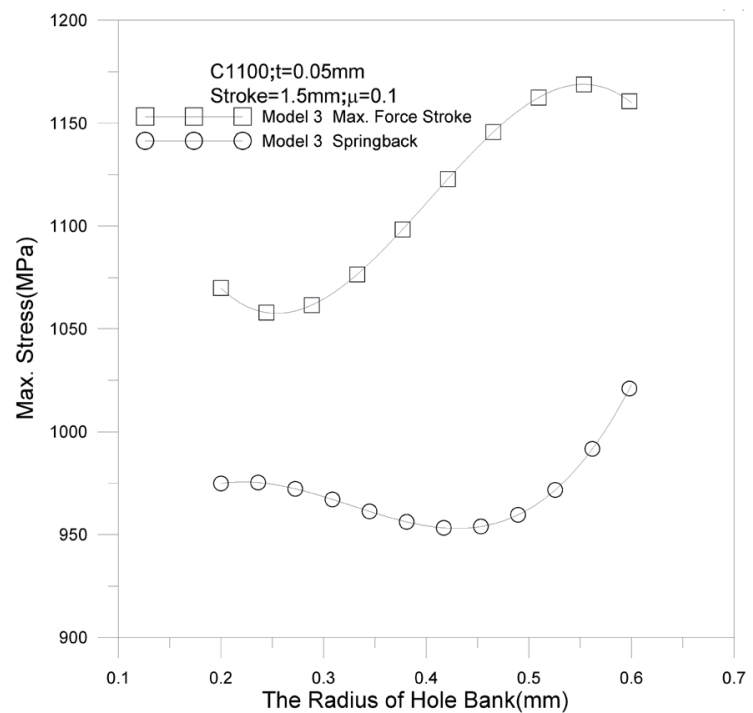

(c)

Fig. 9. Relationship between Br and maximum principal stress of sheet: (a) Model 1; (b) Model 2; and (c) Model 3. 


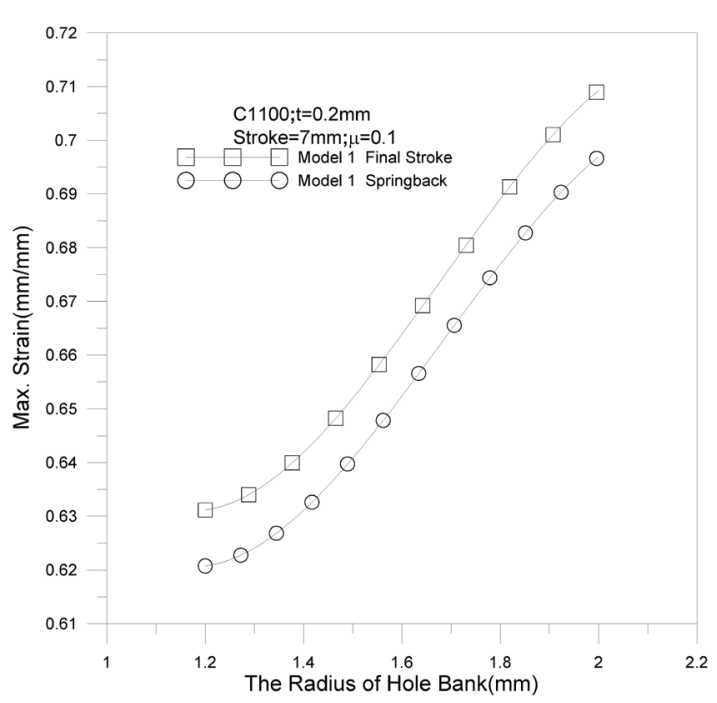

(a)

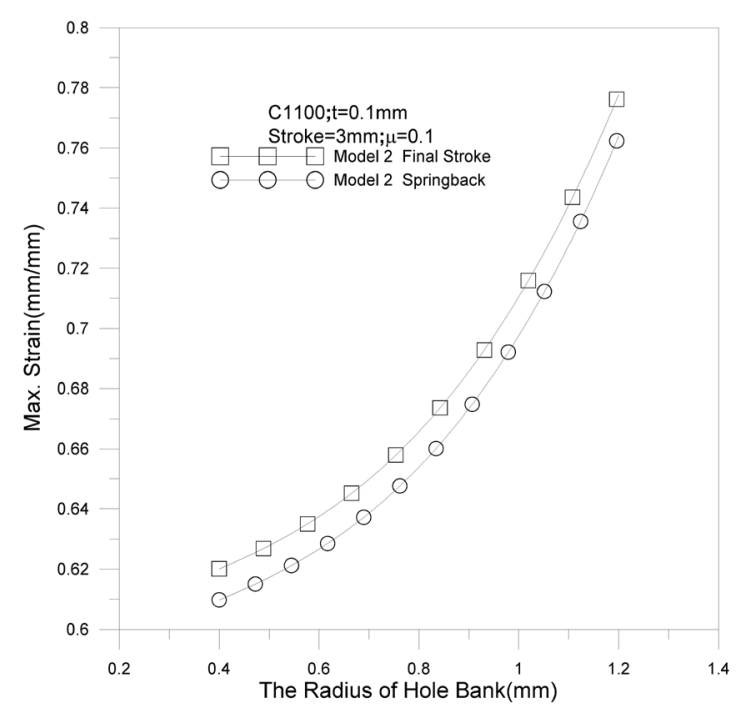

(b)

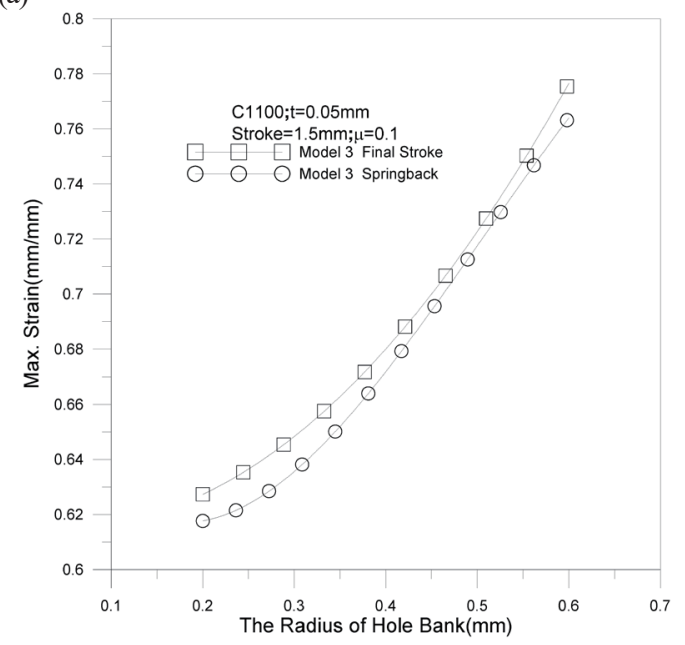

(c)

Fig. 10. Relationship between $\mathrm{Br}$ and maximum principal strain of flange: (a) Model 1; (b) Model 2; and (c) Model 3.

A comparison of the three models shows that miniaturization of the scale allows a greater strain to be induced (Fig. 10). This is mainly because the number of crystal grains in thinner sheets is smaller and better plastic deformation induces more strain. The maximum principal strain increases with $\mathrm{Br}$ simply because the larger Br allows more extensive deformation.

\subsubsection{Relationship between fillet radius and maximum dimension of inner hole of flange}

The maximum dimension of the inner hole $(d)$ is measured for a quarter of the sheet shown in Fig. 1(b). Changing Br may not have a significant effect on the value of $d$ as shown in Fig. 11. 


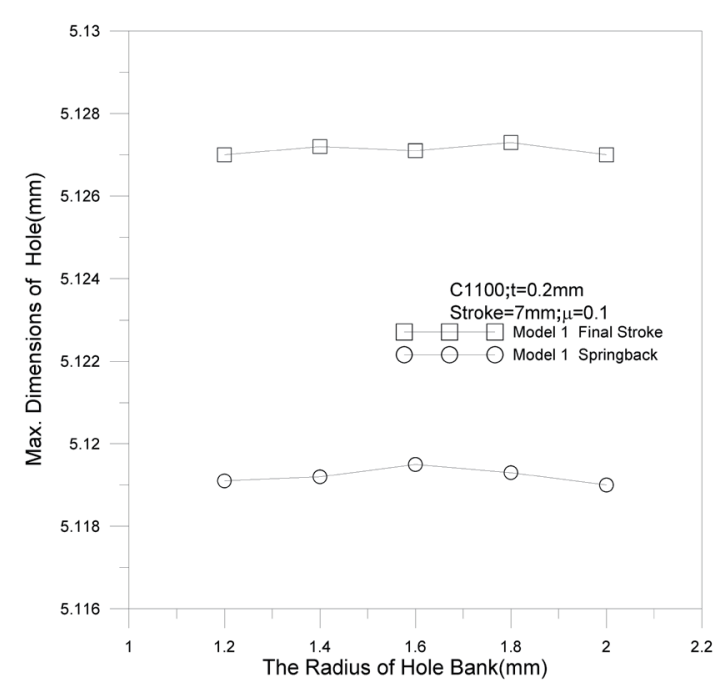

(a)

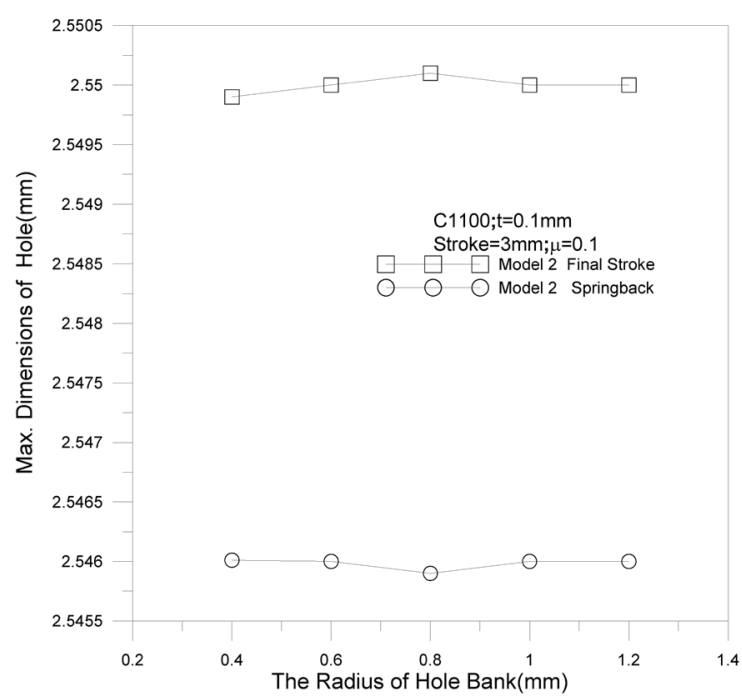

(b)

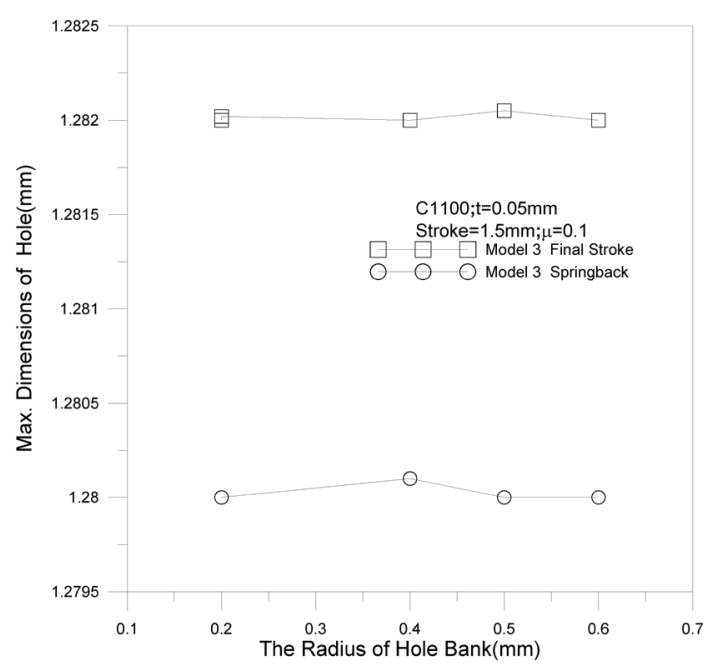

(c)

Fig. 11. Relationship between $\mathrm{Br}$ and maximum dimension of inner hole of flange: (a) Model 1; (b) Model 2; and (c) Model 3.

The main reason is that the dimension and shape of the hole are affected by the geometry of the square punch and are not directly related or influenced by Br. The average values of the maximum dimension of the inner hole of the flange for Models 1-3 are 5.1272, 2.5501, and $1.2821 \mathrm{~mm}$, respectively.

\subsubsection{Relationship between fillet radius and flange height}

The results show that the flange height increases with the fillet radius of the hole. This is because a better forming shape allows the production of a higher flange, as shown in Fig. 12. After unloading, the flange rebounds to slightly reduce its height. 


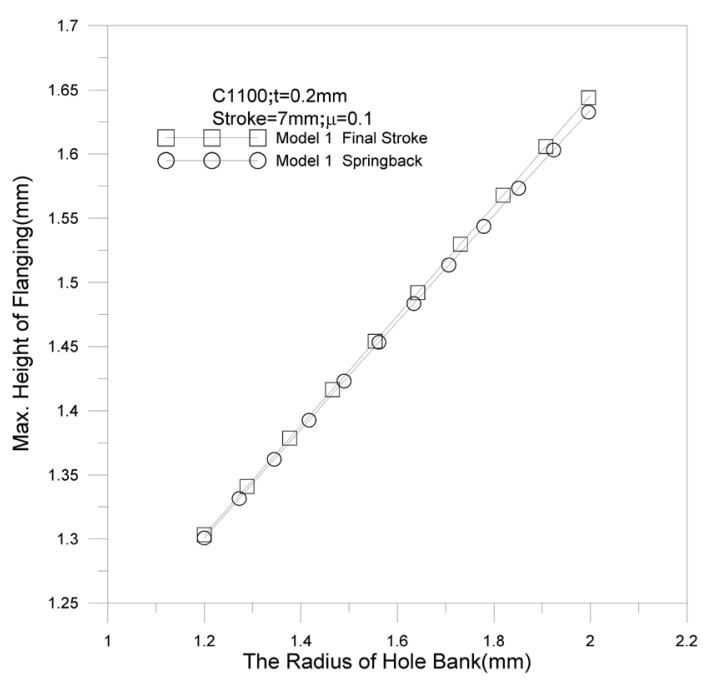

(a)

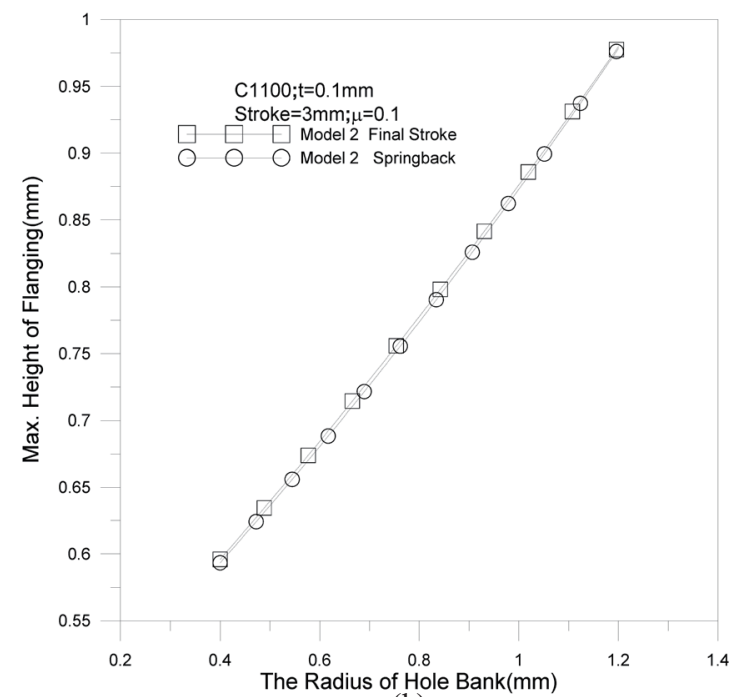

(b)

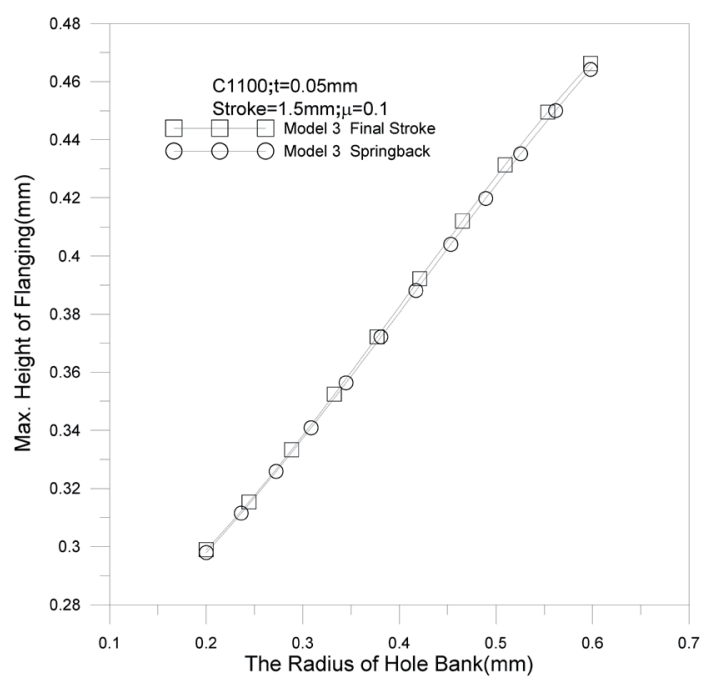

(c)

Fig. 12. Relationship between $\mathrm{Br}$ and flange height: (a) Model 1; (b) Model 2; and (c) Model 3.

\section{Conclusions}

The 3D finite element analysis program used in this study is based on finite deformation theory and an ULF. Dynaform software and the LS-DYNA program were employed for the analysis, and scale factor correction of the influence of the fillet radius of a micro square hole on thin sheets was performed and analyzed. Furthermore, a new microscopic elastoplastic material model was established to modify the influence of the scale effect on micro-thin sheets. The accuracy of this method involving the finite element analysis of elastoplastic deformation was confirmed through simulation and experiments, allowing us to accurately analyze the complete stretch forming process of a flange with a micro square hole. It was found that the minimum 
thickness of the formed flange decreases slightly with increasing $\mathrm{Br}$ for copper sheets in the drawing process. The average values of the minimum thickness for Models $1-3$ in this study are $0.1415,0.071$, and $0.03485 \mathrm{~mm}$, respectively. The maximum principal stress/strain and the flange height increase as $\mathrm{Br}$ increases. Moreover, changing $\mathrm{Br}$ does not have a significant effect on the dimension of the square hole. Using finite element analysis, it is possible to accurately analyze the complete deformation process of flange drawing, obtain the comprehensive deformation history, and predict the forming problems that will occur in the actual drawing process.

\section{Acknowledgments}

This research was funded by the Ministry of Science and Technology of Taiwan (grant number MOST 107-2221-E-167-006).

\section{References}

1 I. Aminzahed, M. M. Mashhadi, and M. R. V. Sereshk: Mater. Sci. Eng. C Mater. Biol. 71 (2017) 685.

2 T. C. Chen and C. M. Hsu: Key Eng. Mater. 626 (2015) 402.

3 W. Phanitwong and S. Thipprakmas: Metals 10 (2020) 870.

4 K. Bouchaâla, M. F. Ghanameh, M. Faqir, M. Mada, and E. H. Essadiqi: Heliyon 7 (2021) e06662.

5 K. F. Su, H. C. Chen, and H. R. Lin: Stamping Guidance (Chuan-HXwa, Taipei, 1991).

6 Y. J. Dai and Y. T. Hsu: Press Processing and Die Design (Tung-Chiao, Taipei, 1981).

7 M. Kawka and A. Makinouchi: J. Mater. Process. Technol. 50 (1995) 105.

8 D. K. Leu: Int. J. Mech. Sci. 38 (1996) 917.

9 H. Takuda and N. Hatta: Mater. Sci. Eng. 242 (1998) 15.

10 T. Kumagai, H. Saiki, and Y. Meng: J. Mater. Process. Technol. 89-90 (1999) 51.

11 M. J. Worswick and M. J. Finn: Int. J. Plast. 16 (2000) 701.

12 Y. M. Huang and K. H. Chien: J. Mater. Process. Technol. 113 (2001) 720.

13 D. K Leu, T. C. Chen, and Y. M. Huang: J. Mater. Process. Technol. 88 (1999) 134.

14 R. M. McMeeking and J. R. Rice: J. Solids Struct. 11 (1975) 601.

15 L. Peng, F. Liu, J. Ni, and X. Lai: Mater. Des. 28 (2007) 1731.

16 F. Liu: Study on the Key Technology of Micro-forming Process, Postdoctoral Thesis: Shanghai Jiao Tong University, Shanghai, China (2006).

17 J. T. Oden and E. B. Pries: ASME J. Appl. Mech. 50 (1983) 67.

18 M. J. Saran and R. H. Wagoner: ASME J. Appl. Mech. 58 (1991) 499. 